\title{
A Study and Performance Analysis of AODV, DSR and GSR Routing Protcols in VANET
}

\author{
Sushil Kumar \\ Computer Science \& Engineering Dept. \\ PEC University of Technology \\ Chandigarh, India
}

\author{
Sudesh Rani \\ Computer Science \& Engineering Dept. \\ Assistant Professor, PEC University of Technology \\ Chandigarh, India
}

\begin{abstract}
VANET is an emerging technology which has taken enormous attention for researcher in the past few years. But due to its high mobility and frequent disconnected characteristics, it's very tough for routing protocols to disseminate data for wireless communication. No single protocol for VANET is able to meet every traffic scenario. Therefore, design of routing protocol is important aspect for efficient data dissemination in any kind of ad-hoc networks. So, we need to analyze the performance of each routing protocols for better routing. This paper present the comprehensive study and performance analysis of AODV, DSR and GSR routing protocols in VANET for vehicle-to-vehicle and vehicle-to-roadside communication.
\end{abstract}

\section{General Terms}

Intelligent Transportation System, Challenges, GPS ,

Communication, Performance

\section{Keywords}

VANET, ROUTING protocols, PARAMETERS, WIRELESS

\section{INTRODUCTION}

For communication between vehicles by wireless technology in Vehicular Ad-hoc Networks (VANET) is a challenging task due to their dynamic nature. VANET is a form of mobile ad hoc wireless networks (MANET) which is a component of Intelligent Transportation Systems (ITS). VANET provide wireless communication between vehicle to vehicle and vehicle to roadside for efficient data transmission. In vehicle to vehicle communication $(\mathrm{V} 2 \mathrm{~V})$, vehicles are equipped with common range to communicate among themselves. But, in vehicle to roadside (V2R) vehicles communicate through enabled access points on the network which causes a lot of additional overhead and delay [1]. The main advantage of vehicular ad-hoc network over cellular network based approach is rapid deployment, robustness, flexibility etc. In VANET, the purpose of V2V and $\mathrm{V} 2 \mathrm{R}$ is that $\mathrm{V} 2 \mathrm{R}$ provides better service sparse networks and long distance communication, whereas $\mathrm{V} 2 \mathrm{~V}$ communication provide direct communication for small to medium distance and at locations where roadside side access points are not available. It is emerging as a new technology to integrate the capabilities of new generation wireless networking to the vehicles.

Similar to Mobile ad-hoc network, VANETs should not require any special kind of hardware infrastructure to control network stability. They require that communication nodes (running inbuilt protocols) execute functions normally which is handled by routers. As if we increase the number of highly mobile nodes, the limitations of controlling such kind of networks become impossible. Thus, one would like to find the volatile topology of the vehicular nodes some properties which remain stable for relatively some periods of time and use them for forming useful protocols. In Vehicular ad-hoc network, each vehicle take on the role of sender, receiver and router to broadcast information to the vehicular network or transportation agency, which then uses the information to ensure that whether the road is traffic free or not. For communication to occur between vehicles and roadside units (RSUs), vehicles must equipped with some sort of radio interface or Onboard Unit (OBU) that enables short-range wireless ad hoc networks to be formed [2]. Here, vehicles must also be fitted with hardware that permits detailed position information such as Global Positioning System (GPS) or a Differential Global Positioning System (DGPS) receiver to facilitate communication.

The inter-vehicle communication configuration (Figure 1) uses multi-hop multicast/broadcast to transmit traffic related information over multiple hops to a group of receivers. There are two types of message forwarding in inter-vehicle communications: naïve broadcasting and intelligent broadcasting. In naïve broadcasting, vehicles send broadcast messages after a certain pre-defined period of time regularly. After receiving the message, the vehicle ignores all the messages which come from a vehicle or vehicles behind it. If the message comes from a vehicle in front, the receiving vehicle sends its own broadcast message to vehicles behind it.

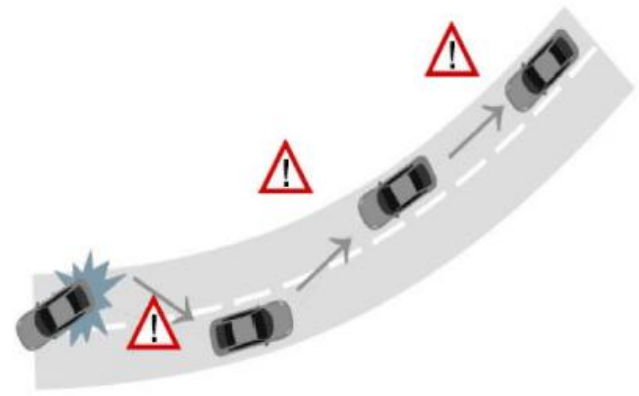

Figure 1 Inter-vehicle communication

The limitation of native broadcasting method is that large numbers of broadcast messages are generated, therefore, increasing the risk of message collision resulting in lower message delivery rates and increased delivery times [3].

The vehicle-to-roadside communication configuration (Figure 2) represents a single hop broadcast where the roadside unit sends a broadcast message to all equipped vehicles in the vicinity. Vehicle-to-roadside communication configuration provides a high bandwidth link between vehicles and roadside units. The roadside units may be placed within specified amount of range, enabling high data rates to be maintained in heavy traffic. The roadside unit will periodically broadcast a message containing the speed limit and will compare any geographic or directional limits with vehicle data to determine if a speed limit warning applies to any of the vehicles in the vicinity. If a vehicle violates to avoid the desired speed, a message will be delivered to the 
vehicle in the form of an auditory or visual warning, requesting that the driver reduce his speed.

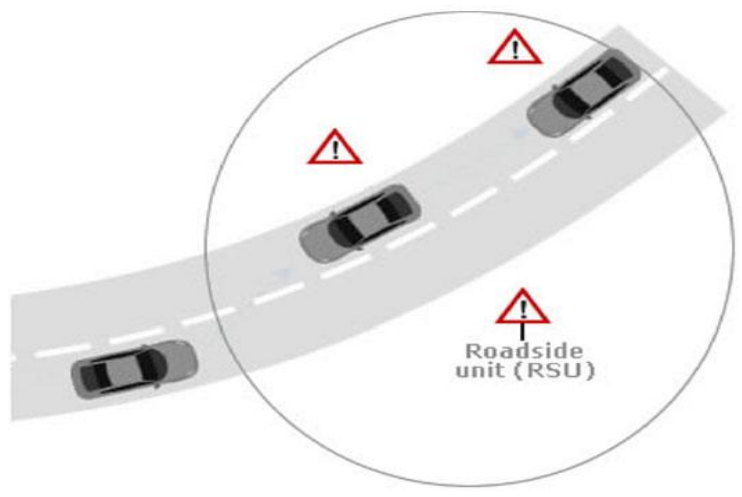

Figure 2 Vehicle-to-Infrastructure communication

A VANET typically has little or no infrastructure. It uses the new technology Dedicated Short Range Communication i.e DSRC for vehicles to communicate. It consists of a number of nodes working together to monitor a region to obtain data about the environment. The subtype of Vehicular Ad-hoc Network is intelligent vehicular ad-hoc network (InVANET). Intelligent vehicular ad hoc networks (InVANETs) use WiFi IEEE $802.11 \mathrm{p}$ (WAVE standard) and WiMAX IEEE 802.16 for efficient data distribution or message passing between vehicles with high mobility. To reduce this sort of problem effective measures such as media communication between vehicles can be enabled to track automotive vehicles (like BMW, AUDI). Intelligent VANET is not used to replace current mobile (cellular phone) communication standards which is very high costs link. The advantage of InVANET is that fewer nodes can be deployed with lower network maintenance and management cost.

The Network design of VANET can be classified into three categories [4]:

a) Pure cellular/WLAN

b) Pure ad-hoc

c) Hybrid

In this paper, we primarily focus on analysis part of various existing routing protocols in VANET.

\section{BACKGROUND}

\subsection{Network Architectures:}

As showed in Figure 3 [5], routing protocols in vehicular networks is based upon three architectures:

2.1.1. Vehicle-to-Vehicle (V2V): This is vehicle to vehicle communication architecture where collection, aggregation and transmission of data are done. This architecture is very effective for faster delivery of messages directly between vehicles within network.

2.1.2. Vehicle-to-infrastructure (V2I): This is vehicle to infrastructure wireless architecture. This structure uses technologies like Global System for mobile communication, WiMax etc. for distribution of information from nodes.

2.1.3. Hybrid: This is combination of both Vehicle-tovehicle and Vehicle-to-infrastructure architectures. Hybrid architect provide better opportunity to make analysis regarding performance of routing protocols.

All the above architecture follow different routing strategy for different communication environments.

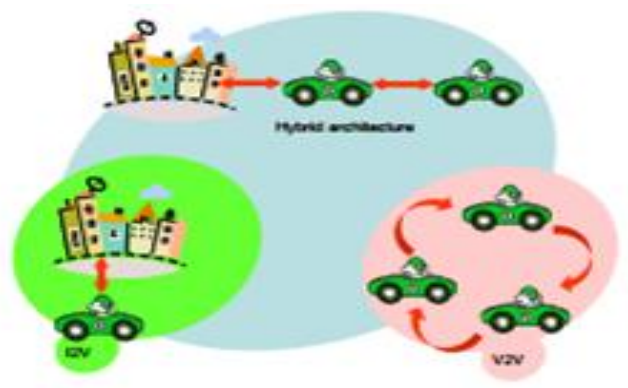

Figure 3 Vehicular communication architectures

\subsection{Various Characteristics in VANET:}

There are so many unique characteristics in vehicular ad-hoc networks which make it different from mobile ad-hoc network. Due to uniqueness in every single characteristics of VANET, it's very tough and challenging for any user to design routing protocols for different scenario (city or highway).

2.2.1. High Mobility: This is major issue in designing any kind of routing protocol is that vehicles have to move at different parts of Metropolitan Area Network (MAN) at high speed for communication. So, mobility is high in this kind of network.

2.2.2. Frequent Disconnection: As we know movement of vehicles is very high in sparse network during day hours. So due to high dynamic topology and change in the network scenario will result into disconnection

2.2.3. Mobility pattern: The mobility structure or behavior of vehicles are depend upon various aspects such as traffic environment, structure of roads, speed and position of vehicles and driving behavior of the driver.

2.2.4. Too many hops: For efficient data distribution in VANET, many hops can be wirelessly connected to each other. So its not easy for vehicles to communicate when two vehicles are requesting for same data packet.

2.2.5. Inbuilt Storage: Traditionally, vehicles was equipped with limited battery power and storage capacity but now days vehicles comes up with unlimited storage capacity which should have enough computing power for effective communication

2.2.6. Communication environment: In VANET, vehicles have to communicate among different environment such as city and highway. In city environment buildings, trees and other objects make tough for vehicles to communicate freely. But in highway these things are not present. Vehicles have to communicate openly. So the routing strategies for both the cases will be different.

\section{ROUTING PROTOCOLS}

Routing of data between vehicles is the challenging task when we working in vehicular ad-hoc networks. Due to its high speed, mobility pattern and changing communication environment performance analysis of any routing protocol is not easy because no single routing protocol will meet all the traffic scenarios. The routing protocol of VAENT can be classified into two main categories i.e Topology based routing protocols and Position based routing protocols. 


\subsection{Ad-hoc On Demand Distance Vector Routing Protocol (AODV)}

The Ad hoc On Demand Distance Vector routing protocol [6] is topology based routing protocol which uses shared link for transferring packet from source to destination in specific manner. This protocol is totally based on shortest path. The main objective of this protocol is to keep the information of neighbor nodes in form of table. Here, every node has to maintain its route table for effective communication. When any changes occur in network each node has to update its routing table with requiring periodic flooding. In this protocol flooding require only when it is demanded. When a node want to send the data it automatically establish a route from source to destination. This protocol works with both unicast and multicast routing. When source wants to send the data to destination through AODV it first find the destination sequence number (DesSeqNum) for transmission which makes it different from other routing protocols.

\section{Advantages:}

- Provide efficient path from source to destination because of using sequence number.

- Efficient memory utilization.

- Reduce route duplicity.

- AODV responded very quickly whenever there is link failure in the network.

- It can be also used for large scale communication purpose especially in Intelligent Ad-hoc Networks (InVANET).

\section{Disadvantages:}

- Require more time to establish connection setup and start the initial routing approach.

- Additional overhead when there is multiple route reply in the network.

- Bandwidth utilization is too much due to periodic beaconing.

- Inconsistency and redundancy if nodes contain old entries in their routing table.

\subsection{Dynamic Source Routing (DSR) Protocol}

This protocol works in two phases ie. First is route discovery and second is route management [7]. DSR utilize source routing address and follow the active path for routing. In DSR, each packet require to carry some address from source to destination, so overhead amount carried out by a packet will leads to consume more bandwidth. Therefore, this kind of protocol is not suitable for large network. Here, sender makes lists of route by finding each 'hop' through address of next node which to send to data on receiver side.

\section{Advantages:}

- It does not require any periodic update of routing information.

- To find routes between different nodes, very less overload on the network.

- Beacon free protocol.

- Low mobility pattern.

- Multiple routes in their route cache.

- DSR uses cache for faster dissemination of data which will reduce some load on the network.

\section{Disadvantages:}

- Not able to repair breakage links.

- Not suitable when too many nodes communicate within the network.

- Due to high mobility pattern, unnecessary flooding will overload the network

\subsection{Geographic Source Routing (GSR) Protocol:}

This protocol for vehicular networks works very well in city environment where traffic density is high during day hours but less in night hours. GSR is combination of position-based with topology based routing. This algorithm first selects the path and finds the shortest distance using Dijkstra algorithm. GSR uses Reactive Location Service (RLS) [8] to get the destination address. This RLS provide best route for sender to send the packet. The basic routing architecture of GSR is shown in figure 4 which follows top-down approach for routing. Application layer provide VANET applications to interact with user and other layers for communication. Transport layer uses protocols like User datagram protocol (UDP) and Transmission control protocol (TCP) for congestion control in VANET. Network layer provide route to the data by using RLS. The Last layer i.e Physical layer provide wireless communication that is working on IEEE standard 802.11a, 802.11b, 802.11g.

In order to avoid many effects during transmission of data such as hidden station problem, broadcast storm problem, flooding etc., many strategies have been implemented for RLS [9].

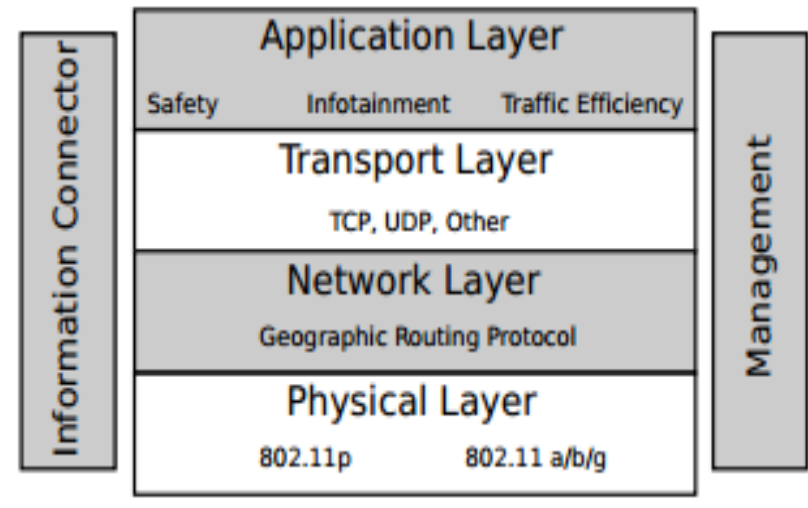

Figure 4 Geographic Routing architecture

\section{Advantages:}

- Provide good packet delivery ratio then AODV and DSR.

- More Scalability as compared to AODV and DSR.

Disadvantages:

This protocol is not suitable in sparse network where number of nodes for packet transmission is less.

GSR should have high overhead on the network due to the uses of beacon i.e hello message as control.

\section{SIMULATION AND RESULTS}

This simulation study basically presents the map based performance of Ad-hoc On Demand Distance Vector Routing (AODV), Dynamic Source Routing (DSR) and Geographic Source Routing (GSR). Simulation can be done using famous simulation tool NS-2 [10]. This section of paper presented the performance based on some performance parameters. Some common simulation parameters are: 
- Packet delivery rate

- Latency

- Bandwidth consumption

- Avg. number of hops

The simulation of the proposed algorithm was based on NS2. The transmission range was set to $500 \mathrm{~m}$. The simulation has been done with multiple nodes. Table 1 show the simulation carried out in NS2 for 75 nodes.

Table 1 Parameters used in NS2

\begin{tabular}{|l|l|}
\hline Channel type & Channel / Wireless channel \\
\hline Propagation model & $\begin{array}{l}\text { Propagation/ Two way } \\
\text { Ground }\end{array}$ \\
\hline MAC & Mac/ 802.11 \\
\hline Antenna & Bidirectional \\
\hline No of nodes & 75 \\
\hline Transmission range & 500 \\
\hline
\end{tabular}

The performance graph of various general simulation parameters is shown in figure $5,6,7,8$.

\subsection{Packet Delivery Rate}

The ratio of the number of delivered data packets to the destination. The greater value of packet delivery ratio means the better performance of the protocol.

The packet delivery rate of GSR is much better than AODV and DSR with respect to distance. GSR provide better packet delivery rate as shown in figure 5 when its transmission range is 500 .

\section{Avg. Delivery Rate}

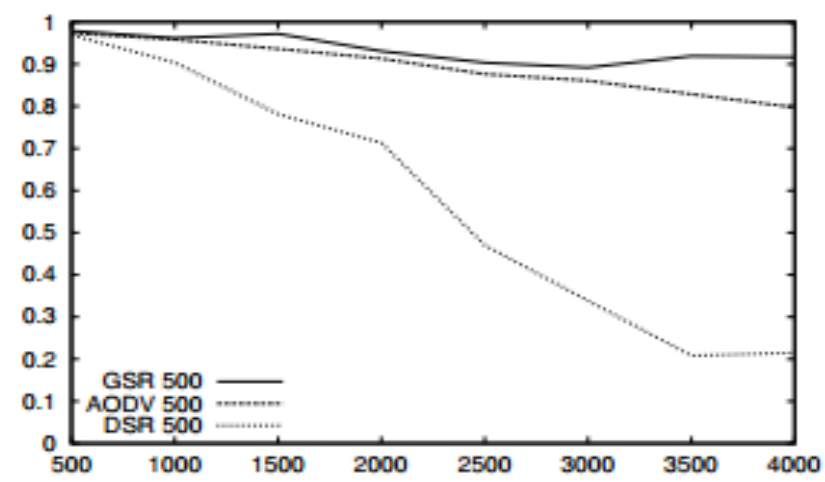

distance $[\mathrm{m}]$

Figure 5 Performance analysis using packet delivery rate Vs distance

But when its range reduced to 250 there are many chance of drop in packet delivery rate.

\subsection{Latency}

The second parameter is latency. Latency is a time interval between the stimulation and response, or, from a more general point of view, as a time delay between the cause and the effect of some physical change in the system being observed As far as latency for first packet transmission is concerned it is observed that both DSR and GSR (As shown in figure 6) have similar with plus factor in favor of DSR. The latency is higher in case of
AODV with respect to both DSR and GSR because of the usage of ring search technique.

\section{Avg. Latency of First Delivered Packet}

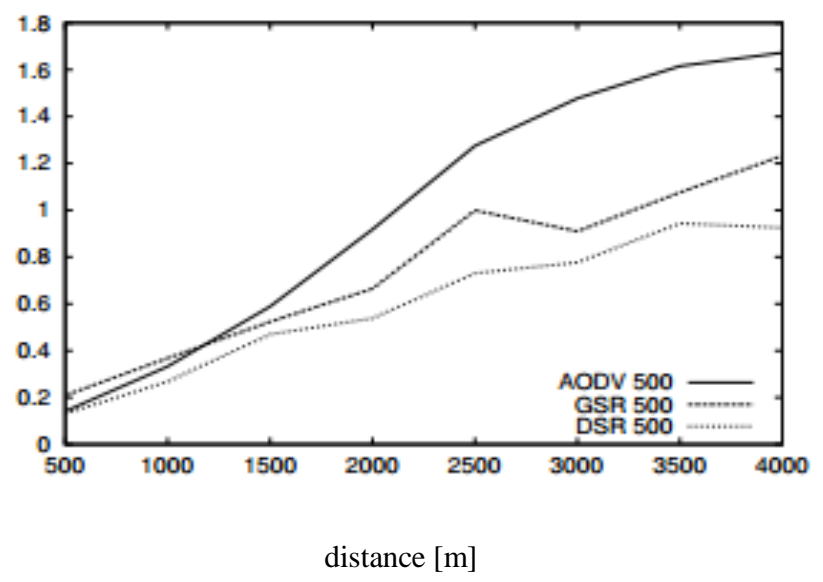

Figure 6 Latency Vs connection

\subsection{Total Bandwidth Consumption}

Another parameter which provide better in analyzing the performance of AODV, DSR and GSR is Total bandwidth consumption. As shown in figure 7 the bandwidth consumption keeps on increasing if we increase the distance because AODV uses ring search method for finding route. The bandwidth consumption according to this figure is very low in AODV with respect to other two routing protocols.

Avg. Total Bandwidth

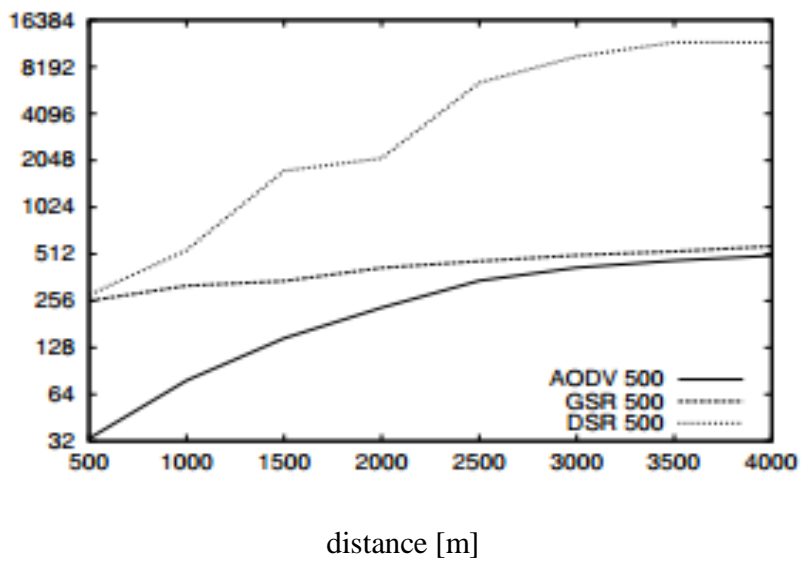

Figure 7 Total bandwidth utilization Vs distance

Therefore, more bandwidth consumption will lead to large volume of energy to be consumed. So distance between the communication nodes should be as less as possible for better routing.

\subsection{Average number of Hops}

In DSR what happened is whenever a route is broken, a node have to drop the packet. Then the dropped packet is not recovered. GSR has some packet recovering strategies. This parameter described about the total number of hops used is totally depending on the distance. More distance requires large number of hops for routing. 
Avg. Number of Hops

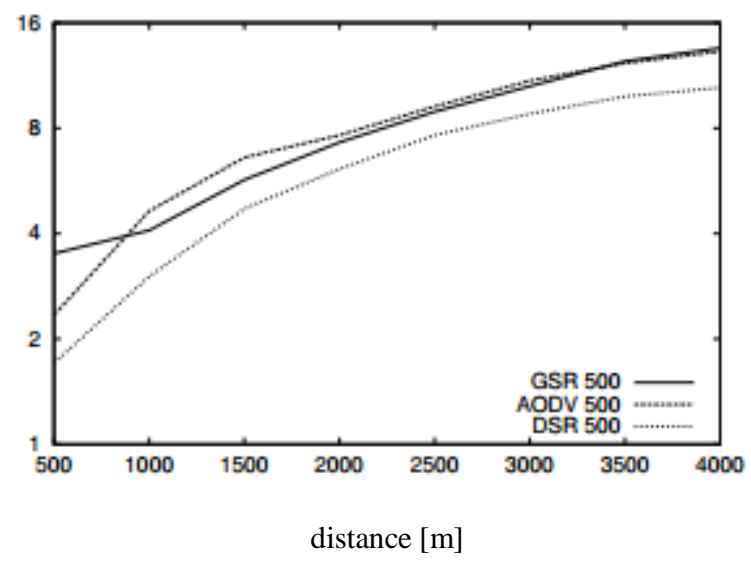

Figure 8 No of hops Vs distance

\section{CONCLUSION AND FUTURE WORK}

In this paper we presented the basic study of routing protocols such as AODV, DSR and GSR for vehicular ad-hoc networks. The comprehensive study of these protocols showed that no single routing protocol will meet the all the traffic requirements. The performance of these protocols has been analyzed using simulation tool. Simulation results showed that performance of these protocols varies with distance, range and speed.

This work can further be extended by performing simulation with different traffic scenarios and by introducing some more parameters for performance evaluation. This will provide us better understanding about routing in vehicular ad-hoc networks.

\section{ACKNOWLEDGMENTS}

This work was supported by Cyber Security research lab and CL 14 at PEC University of technology, Chandigarh (Formerly Punjab Engineering college) and also my guide who is currently the coordinating Computer Science and Engineering department at PEC University, Chandigarh.

\section{REFERENCES}

[1] Sushil Kumar and Sudesh Rani, "A study and comparsions of Cluster-based data dissemination protocols in VANET" March 2014.

[2]Stampoulis,A. \& Chai, Z. (2007), "A survey of security in vehicular networks." http://zoo.cs.yale.edu/ ams257/projects/wireless-survey.pdf (accessed: May 29, 2010).

[3] Bickel,G.(2008), "Inter/intra-vehicle wireless communication"

http://userfs.cec.wustl.edu/ gsb1/index.html\#toc (accessed: May 29, 2010).

[4] "Survey of Routing protocols in Vehicular Ad-hoc Networks," Kevin C. Lee, Uichin Lee, Mario Gerla, Advances in Vehicular Ad-hoc Networks: Developments and challenges, IGI Global, Oct, 2009.

[5] Ismail Salhi, Mohamed Oussama Cherif, and Sidi Mohammed Senouci, "A New Architecture for Data Collection in Vehicular Networks", IEEE ICC, 2009.

[6] Perkins, C.; Belding-Royer, E.; Das, S, “Ad-hoc On-Demand Distance Vector (AODV) Routing”, July 2003.

[7] Johnson, D. B. and Maltz, D. A, "Dynamic Source Routing in Ad Hoc Wireless Networks, Mobile Computing" T. Imielinski and H. Korth, Eds., Ch. 5,1996, pp. 153-81.

[8] W. Kiess, H. Fussler, J. Widmer, and M. Mauve, "Hierarchical location service for mobile ad-hoc networks," SIGMOBILE Mob. Comput. Commun. Rev., vol. 8, no. 4, pp. 47-58, 2004.

[9] M.Kasemann, H.Fubler, H. Hartenstein and M.Mauve, "A Reactive Location Service for Mobile Ad-hoc Networks", Technical Report, Department of Computer science, University of Mannheim, Nov. 2002.

[10] The ns-2 network simulator. http://www.isi.edu/nsnam/ns/. 